\title{
Replik zur kritischen Stellungnahme von Johannes G. Schmidt zum wissenschaftlichen Ärztekongress VegMed 2012 (Forsch Komplementmed 2013;20:469)
}

\author{
Christian Kessler Markus Keller Andreas Michalsen
}

Abteilung für Naturheilkunde, Immanuel-Krankenhaus Berlin, Deutschland

Zunächst wird eine allseits bekannte und damit doch triviale Kritik an epidemiologischen Studien benannt, das sogenannte Confounding Bias. Dies trifft selbstverständlich für alle epidemiologischen Kohortenstudien in gewissem Maße zu. Da allerdings Ernährungsforschung nur in sehr begrenztem Maße (aufgrund der Adhärenz) mit randomisierten kontrollierten Studien und per se nicht mit verblindeten Studien betrieben werden kann, ist die Evidenz aus Kohortenstudien eine ausgesprochen wichtige und gute Evidenzquelle, vor allem wenn es sich (wie zum Beispiel bei der seit 2002 laufenden Adventist Health Study II (www.llu.edu/public-health/health/early_findings. page) mit über 90000 Teilnehmern!) um Studien von beachtenswerter Größenordnung handelt. Erinnert sei in diesem Zusammenhang auch daran, dass die Evidenz für die Schädlichkeit des Rauchens initial auch nur aus epidemiologischen Studien abgeleitet werden konnte.

Natürlich gibt es auch korpulente Vegetarier. Klassisch ist auch die Erwähnung des kettenrauchenden Opas, der sich mit 95 Jahren bester Gesundheit erfreut. Gerade auf diesem Niveau sollten aber medizinisch-wissenschaftliche Diskussionen nicht geführt werden. Zu beachten ist ferner, dass die Evidenz der Epidemiologie sich nicht nur auf mögliche lebensverlängernde Effekte bezieht: Die vorliegende Evidenz zeigt konsistent, dass eine vegetarische oder vegane Ernährungsweise bei fast jeder untersuchten chronischen Erkrankung zu einer Reduktion der Morbidität oder Mortalität geführt hat. Es gibt hingegen keinerlei Evidenz, die Fleischverzehr diesbezüglich positiv bewertet. Im Gegenteil - jede Reduktion des Fleischkonsums verringert das Erkrankungsrisiko: Selten-Fleischesser (maximal eine Fleischmahlzeit pro Woche) schneiden besser ab als Fleischesser (mehr als eine Fleischmahlzeit pro Woche); Fischesser (kein Fleisch-, aber Fischverzehr) besser als Selten-Fleischesser; und Vegetarier bzw. Veganer (meist) am besten. Darüber hinaus zei- gen auch vegetarische bzw. vegane Ernährungsinterventionen in randomisierten Studien signifikant günstige Effekte auf unter anderem Übergewicht, Diabetes mellitus Typ 2, Bluthochdruck sowie Prostatakarzinom - und führen interessanterweise auch zu einer Verbesserung des psychischen Wohlbefindens.

Im Gegensatz dazu stehen nun die vorgebrachten Postulate der chinesischen Medizin bezüglich ihrer Ernährungslehre ohne jegliche wissenschaftliche Evidenz da. Dass möglicherweise in kalten Klimazonen und nach Hungersnöten (die auch die chinesische Geschichte bis in die 1960er Jahre geprägt haben) nährende Fleischgerichte einen (begrenzt) sinnvollen Platz hatten und als subjektiv angenehm empfunden wurden, ist nachvollziehbar. Die Lebensbedingungen in modernen Gesellschaften sind jedoch völlig anders: Wir leben nicht in Hunger, Kälte und Auszehrung, sondern in (teils extremem) alimentärem Überfluss und mit Bewegungsmangel. Insofern ist der Rekurs auf traditionelle Empfehlungen aus anderen Kulturkreisen im geschichtsfreien Raum und ohne jegliche Evidenz hier wenig hilfreich und leistet keinen konstruktiven Beitrag für eine moderne ernährungswissenschaftliche Diskussion.

Die zunehmende wissenschaftliche Beweislage für den Nutzen vegetarischer Ernährungsweisen sollte auf dem Kongress VegMed dargestellt und kritisch diskutiert werden. Darüber hinaus will VegMed jedoch bewusst über den Tellerrand der medizinischen Evidenz schauen und auch globale Aspekte wie die ökologische, ökonomische und ethische Dimension der vegetarischen Ernährung beleuchten (z.B. Massen- bzw. Intensivtierhaltung, Treibhausgasemissionen, Wasserverbrauch, Welternährung).

Vehement widersprechen möchten wir auch der Äußerung, vegetarische Ernährung sei eine «Mode» in einer urbanen Welt. Unser Planet ist aufgrund des weltweit steigenden Fleischkonsums mit sehr ernsten und massiven Problemen

\section{KARGER}

Fax +497614520714

Information@Karger.com

www.karger.com (c) 2013 S. Karger GmbH, Freiburg

$1661-4119 / 13 / 0206-0470 \$ 38.00 / 0$

Accessible online at:

www.karger.com/fok
Prof. Dr. med. Andreas Michalsen

Abteilung für Naturheilkunde

Immanuel-Krankenhaus Berlin

Am Kleinen Wannsee 5, 14109 Berlin, Deutschland

a.michalsen@immanuel.de 
konfrontiert. Alle seriösen globalen Szenarien und diesen zugrundeliegende wissenschaftliche Daten zeigen, dass unsere Erde mit einer Bevölkerung von 8-10 Milliarden Menschen (Prognose für 2050) ohne Hunger oder Umweltkatastrophen letztlich nur mit einer überwiegend vegetarischen Ernährung vorstellbar ist. Initiativen und Verhaltensweisen, die zur Rettung unseres Planeten beitragen, sind deshalb keine Modeerscheinungen, sondern Notwendigkeiten. Darüber hinaus sind vegetarische Ernährungsweisen historisch in vielen großen Kulturen seit Jahrtausenden dokumentiert und stellen in einigen Weltregionen wie in Indien sogar fast die Haupternährungsform dar.

Schließlich äußert Schmidt, dass Vegetarismus keine Naturheilkunde sei. Tatsächlich ist die vegetarische Form der Vollwerternährung im Rahmen der Lebensreformbewegung entstanden und wird noch heute in naturheilkundlichen Kliniken als Basistherapie betrachtet und eingesetzt. Die vegetarische Ernährung kann deshalb als integraler Bestandteil der europäischen Naturheilkunde eingeordnet werden.
Abschließend ist anzumerken, dass die Berücksichtigung von Konstitution und Verträglichkeit (z.B. von Rohkost) selbstverständlich in jede gute naturheilkundliche Praxis mit vegetarischer Ernährungsausrichtung einfließt. Vegetarische Ernährung und Rohkost sind keinesfalls als Synonyme zu verwenden. Nicht für jeden ist Rohkost in jeder Lebenssituation geeignet; insbesondere der Anteil an der gesamten Kost muss individuell abgestimmt sein. Vegetarische Ernährung erlaubt hier jedoch eine große Bandbreite an «stärkender» und leicht verdaulicher Kost, die alle gängigen Zubereitungsverfahren einbeziehen kann.

Nicht zuletzt sei auf das andere große traditionelle Medizinsystem östlicher Provenienz verwiesen, den Ayurveda. Hier wurden ähnliche Aspekte der Konstitution in einer überwiegend vegetarischen und konstitutionell ausgerichteten Ernährung hochdifferenziert ausgearbeitet. Etwas (subjektiv) Wärmenderes und Stärkenderes als ein gut gewürztes indisches Gemüsecurry gibt es wohl kaum - der Autor sei hiermit herzlich eingeladen, es einmal als Fleischalternative auf dem VegMed-Kongress 2013 auszuprobieren. 